\title{
Growth Feature of Diamond-Like Carbon Films by Various Vacuum Plasma Methods
}

\author{
Zhi-Feng WANG, Bing ZHOU*, Zhu-Bo LIU, Zhi-Yong HE \\ Research institute of surface engineering, Taiyuan University of Technology, Taiyuan 030024, \\ China
}

\begin{abstract}
Keywords: Diamond-like carbon, Vacuum plasma methods, Microstructure, Surface morphology, Optical property.
\end{abstract}

\begin{abstract}
Diamond-like carbon (DLC) films were prepared on silicon substrate using four different vacuum plasma methods: magnetron sputtering (MS), pulse laser ablation (PLA), ion beam deposition (IBD) and pulse cathode vacuum arc evaporation (CVAE). The microstructure, surface morphology and optical properties of the DLC films were investigated by Raman spectroscopy, atomic force microscope (AFM) and UV-visible spectrophotometer. The results showed that the films prepared by different vacuum plasma methods varied in the microstructure. The film prepared by CVAE possessed the smallest grain size of graphite with the highest disordering of Csp2 clusters, while more disordered Csp2 clusters presented in the DLC film due to the high ions energy density during PLA. Meanwhile, the deposition methods mainly affected the size of particles and roughness of the DLC films under the same thickness. The film by PLA shows a quite smooth surface structure with uniform particle size. In terms of the optical properties, the films prepared by magnetron sputtering expressed the best optical performance among the four vacuum plasma methods.
\end{abstract}

\section{Introduction}

Diamond-like carbon (DLC) is a metastable form of amorphous carbon with a mixture of graphitelike $\mathrm{sp}^{2}$ and diamond-like $\mathrm{sp}^{3}$ bonding. DLC films are widely applied as protective coatings, such as mechanical, acoustic, electronic and optical devices and magnetic media because of their excellent properties, including high mechanical hardness, optical transparency, good chemical stability and thermal conductivity [1-3]. In 1971, Aisenberg and Chabot [4] successfully prepared the Diamondlike carbon (DLC) films for the first time. From then on, more and more deposition techniques and methods have been created to improve the comprehensive properties of the DLC films during the past fourty years. Currently, the DLC films are mainly fabricated by the following methods, such as magnetron sputtering (MS) [5,6], pulsed laser ablation (PLA) [7,8], ion beam deposition (IBD) $[9,10]$, and cathode vacuum arc evaporation (CVAE) [11,12]. And the composition, structure and performance are varied with different deposition methods.

RF-magnetron (RF-MS) technology is one of the most popular methods. It displays some additional advantages over other methods, including the higher sputtering rate of graphite and the more content of strong $\mathrm{sp}^{3}$-hybrized carbon bonding when compared to IBD. When it comes to PLA, it is an effective technique for producing DLC films that possess hard and smooth surfaces with good adhesion to substrates. However, there are many disadvantages existing in the process of the thin film deposition when using laser as a sputtering source, such as the high energy consumption, the small deposition area and nonuniform thickness of the films [13]. Compared with other methods, IBD is a low temperature deposition method with a better ion ratio and higher ion energy. Although the process of IBD can be controlled flexibly, it is hard to deposit the thin films on the substrate material with a large area [14]. When referred to CVAE technique, it can export high valence and strong energy plasmas with high ionization rate. In addition to strong adhesion between the substrate and film, CVAE is an effective technique to deposit large area of wearresistance carbon films. However, the graphite target easily produces some large size of graphitic particles due to the effect of high temperature electric arc, which will form the rough surface and greatly reduce the performance of the film. Some other techniques are applied to CVAE to 
eliminate these negative effects, including installing filter pipe, shield plate or supplying pulse source. Somehow, these methods will significantly reduce the deposition rate and the uniform deposition areas.

In this work, we fabricated the DLC films with various vacuum plasma techniques, including magnetron sputtering, pulse laser ablation, ion beam deposition and pulse cathode vacuum arc evaporation. The dependence of microstructure, surface morphology and optical properties of the films was investigated on the preparation method.

\section{Experimental}

DLC films were synthesized on single-crystal silicon (100) and quartz glass substrate using RFmagnetron sputtering (RF-MS) [6], pulse laser ablation (PLA) [7,8], ion beam deposition (IBD) [10] and pulsed cathode vacuum arc evaporation (CVAE) [12]. Graphite targets (99.5\% purity) with different size were used as source materials in the deposition process of four techniques. The deposition devices of different techniques have been illustrated in our previous works. The main deposition parameters of different methods were summarized in table 1 . The substrate was ultrasonically cleaned for $20 \mathrm{~min}$ in acetone, ethanol and deionized water to remove other surface contaminants. At a base pressure of $6 \times 10-4 \mathrm{~Pa}$, the substrate was etched by ion sputtering source for 15 min to clean the oxide layer on the surface. Ar+ energy and ion current density were about 4 $\mathrm{KeV}$ and $\sim 25 \mathrm{~A} / \mathrm{m} 2$, respectively. The bias voltage with different deposition methods is about $300 \mathrm{~V}$. The substrate was cooled to room temperature after sputtering clean. During films deposition the rotation velocity of the sample holder was kept at $2 \mathrm{rev} / \mathrm{min}$. The film thickness was controlled by deposition time and was measured by a step device (Ambios Technology XP-2). The thickness of DLC films in present work was 150 180 nm.

Table 1. Deposition parameters of DLC films by different techniques

\begin{tabular}{|l|l|l|l|}
\hline $\begin{array}{l}\text { Preparation } \\
\text { methods }\end{array}$ & $\begin{array}{l}\text { Graphite target } \\
\text { diameter, } \mathrm{mm}\end{array}$ & $\begin{array}{l}\text { Process } \\
\text { pressure, } \mathrm{Pa}\end{array}$ & Discharge voltage, V \\
\hline RF-MS & 50 & $0.2(+\mathrm{Ar})$ & 1250 \\
\hline PLA & 20 & $2 \times 10-3$ & $\begin{array}{l}\text { Nd: YAG laser, } 355 \mathrm{~nm}, 10 \mathrm{~Hz} \\
\text { and pulse duration of } 7 \mathrm{~ns}\end{array}$ \\
\hline IBD & 80 & $5 \times 10-2(+\mathrm{Ar})$ & 5000 \\
\hline CVAE & 30 & $2 \times 10-3$ & $350 \mathrm{~V}$, pulse frequency $3 \mathrm{~Hz}$ \\
\hline
\end{tabular}

The microstructure of the films was analyzed using a Renishaw inVia Raman spectrometer. Raman spectra were excited with the $514.5 \mathrm{~nm}$ emitting lines from an Ar-ion laser at an incident power of $20 \mathrm{~mW}$. Surface morphology of the films was observed by atomic force microscope (AFM) using a multimode scanning microscope Solver-PRO P47 (NT-MDT) in tapping mode, with a scanning scope of $1 \times 1 \mu \mathrm{m}$ and a scan rate of $1.0 \mu \mathrm{m} / \mathrm{s}$. Optical transmittance in the range of $200 \sim$ $1100 \mathrm{~nm}$ were carried out bya UV-1201 UV-Visible spectrophotometer using a blank substrate as reference.

\section{Results and Discussion}

Raman spectra of DLC films prepared by different plasma techniques are shown in Fig. 1. All spectra display a typical spectra characteristic of amorphous carbon structure: a broad Raman peak in the range of 1100 1800 cm-1 with a shoulder peak at around $1360 \mathrm{~cm}-1$. It can be seen from Fig. $1 \mathrm{a}$ and $\mathrm{c}$ that the DLC films prepared by RF-MS and IBD have a stronger shoulder peak, implying a higher content of sp2-hybridized carbon in the films . Raman spectra of DLC films can be fitted with two Gaussian peaks located at about 1360 and $1570 \mathrm{~cm}-1$, which are assigned to the D peak and $G$ peak, respectively [15]. The $G$ peak is dominated by stretching vibration of all pairs of $\mathrm{sp}^{2}-$ bonded carbon atoms in both aromatic rings and carbon chains. The $\mathrm{D}$ peak is attributed to disorder- 
activated aromatic modes of A1 symmetry and originates from the vibration mode of $\mathrm{sp}^{2}$ carbon clusters only in aromatic rings. Fitting results of Raman spectra are summarized in table 2 . The variation in the intensity ratio of the $\mathrm{D}$ peak to the $\mathrm{G}$ peak (ID/IG), the position and width of the Gpeak can give some information on the ordering, amount and size of $\mathrm{Csp}^{2}$ clusters in the DLC films.

Ferrari and Robertson [15] have studied that the ID/IG ratio varies directly with the in-plane correlation length (La) or grain size of the graphite:

$$
I_{D} / I_{G}=c(\lambda) \square L_{a}^{2}
$$

where the coefficient $c(\lambda)$ depends on the excitation wavelength. This relationship is valid for crystalline sizes smaller than $20 \AA$. According to this equitation ID/IG will increase with increasing ordering degree of graphite clusters or increasing size of $\mathrm{Csp}^{2}$ clusters. From table 2, the calculated results show that DLC films by RF-MS and by CVAE have the largest and the smallest grain size of graphite, respectively. This is well consistent with the variation of the width of the $G$ peak. The broadest $\mathrm{G}$ peak in the DLC film by CVAE indicates the highest disordering degree of Csp ${ }^{2}$ clusters. It should be noted that for purely $\mathrm{sp}^{2}$-hybridized carbon materials, such as graphite and graphene, the $\mathrm{G}$ peak is expected to be at $1580 \mathrm{~cm}^{-1}$ and has a sharper form. In the case of DLC films the position of the $G$ peak is found to shift to low wave-number depending on the amount of tetrahedral bonding $\left(\mathrm{Csp}^{2}-\mathrm{Csp}^{3}\right.$ bonds or $\mathrm{Csp}^{3}-\mathrm{Csp}^{3}$ bonds) $[15,16]$. Therefore the position of the $\mathrm{G}$ peak shifts to a lower wave-number in the DLC film by PLA, indicating more disordered $\mathrm{Csp}^{2}$ clusters present in the films. This may be related to the high ions energy density during PLA, facilitating the formation of $\mathrm{sp}^{3}$ hybridized bonding [17,18].

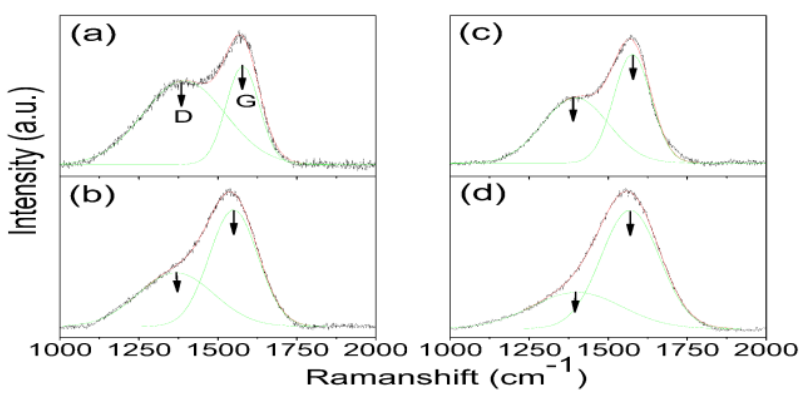

Figure 1. Raman spectra of DLC films prepared by different methods: (a) magnetron sputtering; (b) pulse laser deposition; (c) ion beam deposition; (d) pulse cathode vacuum arc.

Table 2. Gaussian fitting results of Raman spectra, RMS roughness and Eg of DLC films by different preparation methods

\begin{tabular}{|c|c|c|c|c|}
\hline $\begin{array}{c}\text { Preparation } \\
\text { methods }\end{array}$ & ID/IG ratio & $\begin{array}{c}\text { Size of Csp }{ }^{2} \\
\text { cluster, } \AA\end{array}$ & $\begin{array}{c}\text { G-peak } \\
\text { position, }\left(\mathrm{cm}^{-1}\right)\end{array}$ & $\begin{array}{c}\text { G-peak } \\
\text { width, }\left(\mathrm{cm}^{-1}\right)\end{array}$ \\
\hline RF-MS & 2.1 & 19.5 & 1578.2 & 104.7 \\
\hline PLA & 1.04 & 13.8 & 1549.6 & 153.5 \\
\hline IBD & 0.71 & 11.4 & 1576.2 & 118.5 \\
\hline CVAE & 0.47 & 9.2 & 1569.2 & 184.6 \\
\hline
\end{tabular}

Table 3. The RMS roughness and Eg of DLC films by different preparation methods

\begin{tabular}{|c|c|c|}
\hline Preparation methods & RMS, $(\mathrm{nm})$ & Eg, $(\mathrm{eV})$ \\
\hline RF-MS & 1.2 & 0.93 \\
\hline PLA & 0.5 & 1.42 \\
\hline IBD & 0.9 & 1.15 \\
\hline CVAE & 1.7 & 1.28 \\
\hline
\end{tabular}

Surface morphology of DLC films on Si substrate is observed by atomic force microscope, as shown in Fig. 2. It can be seen that column structure containing carbon particles with different size 
distributions present in the DLC films prepared by different techniques. Among of them DLC film by PLA displays a quite smooth surface structure with uniform size (Fig. 2b). DLC film by RF-MS shows fine particle structure but DLC film by CVAE possesses larger particles with a wide size distribution. Root mean square (RMS) roughness of DLC films are listed in table 3. It is obvious that indicates the smallest RMS roughness present in PLA carbon film. These results indicate that deposition process largely affects the surface morphology of DLC films under the same thickness. During the process of CVAE, incident carbon ions/atoms onto the substrate surface have enough energy to achieve the absorption and diffusion along the surface. Moreover in the evaporated particles present some larger size of graphitic particles due to the formation of droplet phase produced by arc discharge, even with the pulse source. Thereby DLC films prepared by CVAE easily form larger particles and rough surface.

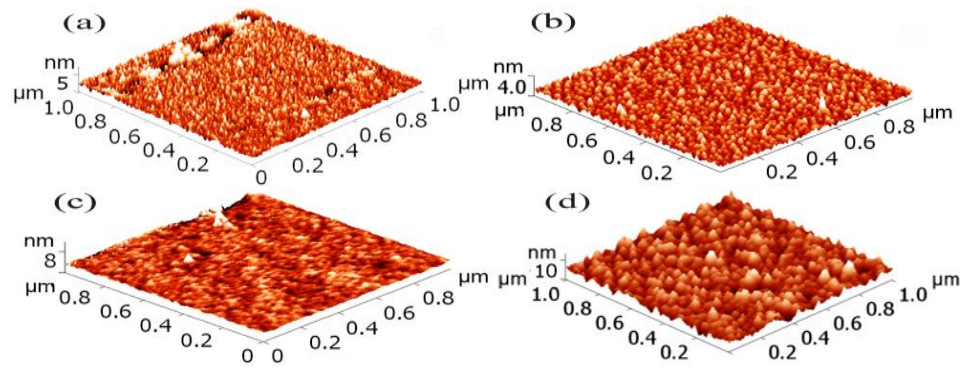

Figure 2. AFM image of DLC films prepared by different methods: (a) magnetron sputtering; (b) pulse laser deposition; (c) ion beam deposition; (d) pulse cathode vacuum arc.

Fig. 3 shows the optical transmittance of DLC films prepared by different techniques in UV-Vis region. It can be seen that the transmittance of the films is $50 \sim 60 \%$ in the wavelength region of 450-1000 $\mathrm{nm}$ and then decreases when the wavelength exceeds a critical value. The critical value varies with the deposition method depending on the structure of DLC films. The optical band gap (Eg) of the DLC films can be determined through the well-known Tauc relation [19]:

$$
(\alpha h v)^{1 / 2}=B\left(h v-E_{g}\right)
$$

Where $\mathrm{B}$ is a constant and $\mathrm{h} v$ is photon energy, $\alpha=-(\ln T) / d$ is the absorption coefficient, $\mathrm{T}$ is the optical transmittance, and $d$ is the film thickness. Fig. 4 shows Tauc plots of DLC films prepared by different methods. The Eg is deduced from the extrapolation of the linear part of the curve at $\alpha=0$, as listed in table 2. It is found that the Eg of DLC film prepared by PLA is $1.42 \mathrm{eV}$ and for RF-MS is $0.93 \mathrm{eV}$, indicating the difference of electronic band structure of carbon films by different plasma techniques. According to the reference [20,21], the optical gap varies in proportion to the $\mathrm{sp}^{3}$ fraction and is controlled by the ordering and distortions of $\pi$ states in $\mathrm{sp}^{2}$ sites and meanwhile, a broad band gap is also induced by the range of clusters size. From Raman analysis, therefore, the increase of band gap for the DLC film by PLA is related to the increase of $\mathrm{sp}^{3}$ fraction, the decrease of the size and ordering of $\mathrm{Csp}^{2}$ clusters compared to RF-MS. This also explained the variation of optical properties of DLC films prepared by IBD and CVAE.

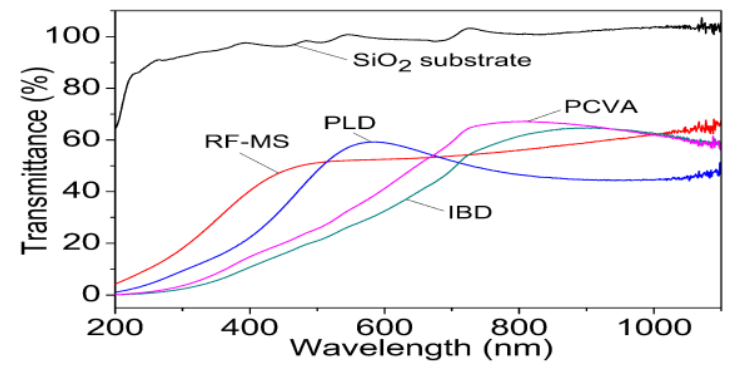

Figure 3. Optical transmittance of DLC films on quartz glass substrate prepared by different techniques. 


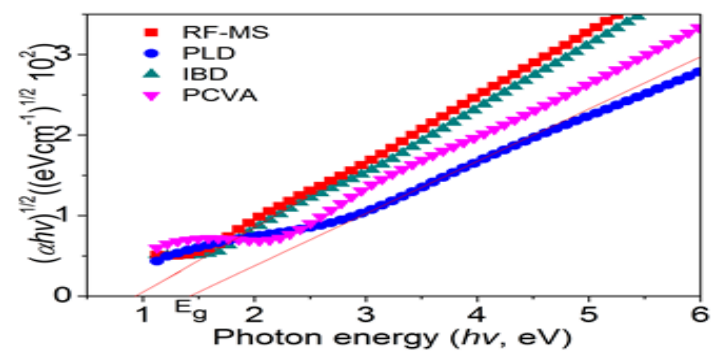

Figure 4. Tauc plot of $(\alpha h v) 1 / 2$ versus photon energy hv for DLC films prepared by different techniques. Solid line is a linear fit in an energy range from $2 \mathrm{eV}$ to $5 \mathrm{eV}$.

\section{Conclusion}

DLC films have been prepared on Si substrate by various vacuum plasma methods, including magnetron sputtering, pulse laser deposition, ion beam deposition and pulse cathode vacuum arc deposition. The differences in structure, morphology and optical property with varying deposition techniques have been studied. The film prepared by MS and CVAE have the largest and smallest grain size of graphite due to the ordering, amount and size of $\mathrm{Csp}^{2}$ clusters in the films. The film prepared by CVAE possesses the highest disordering of $\mathrm{Csp}^{2}$ clusters, while more disordered Csp ${ }^{2}$ clusters present in the DLC film. The difference appears due to the high ions energy density during PLA. Additionally, the deposition process affects the size of particles and roughness of the DLC films in different extent. DLC film prepared by RF-MS shows fine particle structure while DLC film produced by CVAE easily form larger particles and rough surface due to the formation of droplet phase produced by arc discharge. The smallest RMS roughness can be obtained in PLA carbon film. Compared to the four vacuum plasma methods, the films prepared by RF-MS display the best optical performance with the small size and ordering of $\mathrm{Csp}^{2}$ clusters. The optical properties are related to the microstructure and surface morphology of the films and largely depend on the different plasma techniques. According to this study, it can contribute to choose a suitable way to fabricate the film with anticipant and comprehensive properties by a combination of different deposition techniques.

\section{Acknowledgments}

This work was supported by National Natural Science Foundation of China (51502193), Fund Program for the Scientific Activities of Selected Returned Overseas Professionals in Shanxi Province (2015rst), Natural Science Foundation of Shanxi (201601D021057), Qualified Personnel Foundation (tyut-rc201505b) and Special/Young Project Foundation (2014QN036) of Taiyuan University of Technology, Key R\&D program of Shanxi (201603D421035).

\section{References}

1. S. Neuville, J. Surf. Coat. Technol. 206, 4 (2011)

2. J. Robertson, J.Mater. Sci. Eng., R. 37, 4(2002)

3. B. Segura-Giraldo, E. Restrepo-Parra, P.J. Arango- Arango, J. Appl. Surf. Sci. 256, 1(2009)

4. S. Aisenberg, R. Chabot, J. Appl. Phys. 42, 7(1971)

5. S. Zhang, X.L Bui, X. Li, J. Diamond Relat. Mater. 15, 4(2006)

6. B. Zhou, A.V Rogachev, Z.Liu, J. Appl. Phys. 258, 15(2012)

7. S.S Yap, W.O Siew, C.H Nee,J. Diamond Relat. Mater. 20, 3(2011)

8. Z. Liu, A.V Rogachev, B. Zhou,J. Progress in Organic Coatings, 72,3(2011) 
9. H. Hofsäss, H. Binder, T. Klumpp, J. Diamond Relat. Mater. 3, 1-2(1994)

10. Z. Peng, N.N Fedosenko, D.G Piliptsov, J. Проблемы физики, математики и техники, 2, 3(2010).

11. P.C Tsai, K.H Chen, J. Thin Solid Films, 516, 16(2008)

12. B. Zhou, Z. Liu, D.G Piliptsou, J. Appl. Phys. 361, (2016)

13. D. Dijkkamp, T. Venkatesan, X.D Wu, J. Appl. Phys. Lett. 51, 8(1987)

14. C. Weissmantel, K. Bewilogua, D. Dietrich, J. Thin Solid Film. 72, 1(1980)

15. A.C Ferrari, J. Robertson, J. Phys. Rev. B. 61, 20(2000)

16. S. Chowdhury, M.T Laugier, I.Z Rahman, J. Thin Solid Films. 447, (2004)

17. Y.Lifshitz, S.R Kasi, J.W Rabalais, J. Phys. Rev. B. 41, 15(1990)

18. J.J Cuomo, D.L Pappas, J. Bruley, J. J. Appl. Phys. 70, 3(1991)

19. J. Tauc, R. Grigorovici, A. Vancu, J. physica status solidi (b), 15, 2( 1966)

20. Y. Lifshitz, G.D Lempert, E. Grossman, J. Diamond Relat. Mater. 6, 5(1997)

21. J. Robertson, J. Physical Review B. 53, 24(1996) 